\title{
Low wavenumber Raman scattering of cobalt nanoparticles self-organized in 3D superlattices far from surface plasmon resonance
}

\author{
G. Simon, ${ }^{\mathrm{a}, \mathrm{b} *}$ L. Meziane, ${ }^{\mathrm{a}, \mathrm{b}}$ A. Courty, ${ }^{\mathrm{a}, \mathrm{b}} \mathrm{Ph}$. Colomban ${ }^{\mathrm{a}, \mathrm{b}}$ and I. Lisiecki ${ }^{\mathrm{a}, \mathrm{b}}$
}

We report the first observation of low wavenumber Raman scattering from cobalt nanoparticles under $514.5 \mathrm{~nm}$ laser excitation. These $7.4 \mathrm{~nm}$ diameter particles were self-organized in $3 \mathrm{D}$ superlattices, but we assign the Raman signature to vibrations of the individual nanoparticles, according to Lamb's elastic sphere model. We estimate the relative Raman intensity to be around 500 times lower than it would be for silver nanoparticles, but our results demonstrate that surface plasmon-phonon resonance coupling is not mandatory to observe Lamb's modes in metal nanoparticles. Copyright @ 2015 John Wiley \& Sons, Ltd.

Additional supporting information may be found in the online version of this article at the publisher's web site.

Keywords: nanoparticle; cobalt; silver; plasmon

\section{Introduction}

Nanoparticles (NPs) receive considerable attention because their physical and chemical properties are different from those of the bulk material and can be tailored trough size, structural, shape and environmental control. ${ }^{[1,2]}$ Their peculiarity can either be intrinsically related to the size of the particles (the smaller a particle, the higher the curvature and the relative number of symmetrybreaking surface atoms ${ }^{[3-5]}$ ) and/or result from a modification of the surface (chemical reaction, defect gradient, etc.). Gold, copper and silver NPs exhibit a well-defined optical absorption between 400 and

$600 \mathrm{~nm}$, the so-called surface plasmon resonance (SPR), because of the light interaction with a free electron gas at the particle surface. ${ }^{[6-9]}$ These well-defined red or yellow absorptions have been used for millennia to colorglass and glazes. ${ }^{[10,11]}$ Over the last decade, most Raman scattering studies on metallic NPs concerned isolated ${ }^{[12-19]}$ or self-organized ${ }^{[20,21]}$ gold and silver NPs because their surface is chemically stable and choosing a laser excitation close to the SPR allows for a strong enhancement of the Raman signal. Some additional studies were conducted on NPs embedded in a glassy matrix. ${ }^{[10,17,22,23]}$ The vibrational modes expected for the isolated nanoparticles, as predicted by Lamb one century ago ${ }^{[24]}$ to describe earthquake-induced torsional and spheroidal oscillations of the Earth, show below $20 \mathrm{~cm}^{-1}$.25-27] Their observation requires high resolution instruments, namely triple or double holographic grating spectrometers. The sensitivity of these instruments is low, and spectrum collection requires relatively high powers of illumination that can have damaging effect on the material and alter its reactivity.

The use of volume Bragg photonic gratings as optical filters for the Rayleigh line gives access to Stokes and anti-Stokes scattering down to $\sim-10 /+10 \mathrm{~cm}^{-1}$, and their high sensitivity compared to that of double- or triple-grating monochromators allows collecting Raman spectra from poorly resonant or even non resonant materials under low illumination power, without any damage. Thanks to these properties, we present the first observation of low wavenumber Raman scattering from self-organized 3D assemblies of 7.4-nm cobalt NPs and compare their scattering efficiency to that of gold NPs.

\section{Experimental}

\section{Sample preparation and characterization}

Cobalt NPs were synthesized by soft chemical reduction in reverse micelles under a $\mathrm{N}_{2}$ atmosphere. ${ }^{\left[{ }^{28]}\right.}$ The resulting spherical Co NPs were coated with dodecanoic acid and characterized by a mean diameter and size polydispersity of $7.4 \mathrm{~nm}$ and $13 \%$ respectively (Fig. 1a \& 1a'). Studies performed by both electronic diffraction and high-resolution transmission electron microscopy reveal that the as synthesized Co NPs possess a highly disordered polycrystalline structure, with individual crystalline domains (typical size $\leq 1 \mu \mathrm{m}$ ) showing a likely fcc structure. ${ }^{28-30]}$ Because of the presence of the coatingagent, Co NPs are highly stable and neither oxidize, nor coalesce. For this study, 3D assemblies of Co NPs were prepared by slowly evaporating a highly concentrated colloidal hexane solution on a highly ordered pyrolytic graphite substrate (HOPG) maintained at $25^{\circ} \mathrm{C}$. In order to avoid çpbalt oxidation, this step was performed under $\mathrm{N}_{2}$ atmosphere. ${ }^{[3]}$ The optical image presented in Fig. 1b shows that the film obtained after the total evaporation of the solvent is composed of several $2-10-\mu \mathrm{m}$-thick aggregates. The coating agents are not removed after deposition. Previous studies clearly evidenced that Co NPs,

\footnotetext{
* Correspondence to: G. Simon, Sorbonne Universités, UPMC Univ Paris 06, UMR 8233, MONARIS, 4 Place Jussieu, 75005 Paris, France.

E-mail:guilhem.simon@upmc.fr a Sorbonne Universités, UPMC Univ Paris 06, UMR 8233, MONARIS, 4 Place Jussieu,
75005, Paris, France
}

b CNRS, UMR 8233, MONARIS, 4 Place Jussieu, 75005, Paris, France 


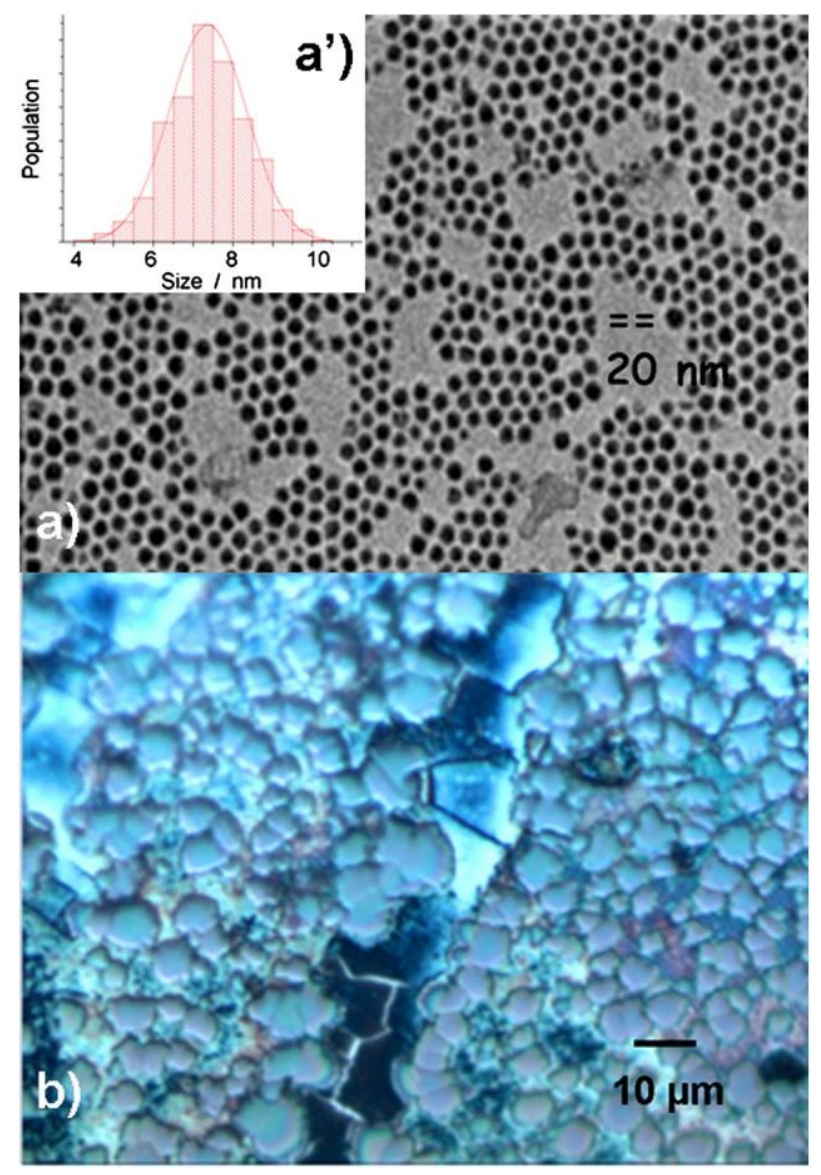

Figure 1. (a) TEM image of a monolayer of cobalt NPs; (a') the corresponding size histogram; (b) optical image of 3D superlattices of Co NPs.

within these domains, are highly ordered in a 3D face-centered cubic (fcc) superstructure which coherence length is a few hundred nanometers. ${ }^{[31]}$ The interparticle gap is equal to $3 \mathrm{~nm}$.

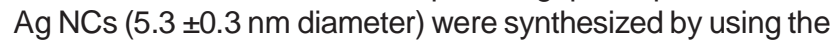
organometallic route described in ${ }^{27} . \mathrm{NO}_{3} \mathrm{AgPPh}_{3}(0.25 \mathrm{mmol})$ was dissolved in $25 \mathrm{ml}$ of ortho-diclorobenzene under nitrogen atmosphere protection. After heating up to $160^{\circ} \mathrm{C}, 500 \mu \mathrm{l}$ of dodecanethiols was quickly injected into the solution under vigorous stirring. The silver salt precursor was then reduced by adding ter-butylamine borane complex $(2.5 \mathrm{mmol}$ dissolved in $15 \mathrm{ml}$ of oDCB). The formation of Ag NCs was evidenced by the colorless solution turning yellow and, eventually, brown red. The reaction mixture was stirred continuously for $1 \mathrm{~h}$ before Ag NPs were washed by precipitation in ethanol and redispersion in toluene. The sample Ag NP assemblies were prepared overnight by evaporating a $200-\mu \mathrm{l}$ volume of the $10^{-3} \mathrm{moll}^{-1} \mathrm{Ag}$ NP solution under nitrogen flux, in a glass beaker in the bottom of which the HOPG substrate was placed. The substrate temperature was fixed at $50^{\circ} \mathrm{C}$. This strategy of NP deposition produces unique well-faceted silver supracrystals ${ }^{[32]}$ larger than 2 microns in dimension.

\section{UV-Visible characterization}

UV-Visible absorption spectra were recorded on a conventional Cary 5000 Varian spectrophotometer. Acquisitions were carried out in hexane for particles in solution, and on a glass plate (made hydrophobic by a 10-nm layer of amorphous carbon) for the supracrystals.

\section{Raman measurements}

A HR800 LabRam setup from Horiba, working with 514.5-nm laser excitation and equipped with BragGrate notch filters from Optigrate (Oviedo, Florida) was used for all Raman measurements. We only used a $\times 100$ microscope objective (Olympus, NA: 0.90), for which the spot waist at the focus point was small enough $(\sim 1 \mu \mathrm{m})$ to fully illuminate a definite surface area of the selforganized supracrystals (Fig. 1b) In order to compare the scattering efficiencies, the laser power was locked around $500 \mu \mathrm{W}$ (at the focus point), and we took careful attention to always focus the same way on the different samples. The spectra have all been recorded under the same optical conditions and with the same incident power. The incoming light was focused in such a way to always optimize the Raman signal. Apart from a constant background subtraction by a straight line when required, all the spectra are raw data.

This low illumination power does not guarantee absence of heating. Optical examination and Rayleigh line broadening must be controlled carefully (fig. S1, Supporting Information).

Polarized measurements were carried out on single focus points using polarizers and $\lambda / 2$ wave-plate accessories to change the configuration and avoid any polarization effect induced by the grating. We thus obtained precise depolarization ratios for the different Raman active modes.

\section{Results and discussion}

Figure 2 shows UV-Visible absorption spectra of cobalt and silver nanoparticles either dispersed (sol) in hexane or self-organized on a glass slide (film) Ag NPs dispersed in hexane display a strong absorption centered at $427 \mathrm{~nm}$, characteristic of the surface plasmon resonance, (SPR) of silver. Despite the red-shift (from 427 to $461 \mathrm{~nm}$ ) and broadening resulting from the interparticle plasmon coupling, ${ }^{[33,34]}$ the resonance remains in the visible range when the particles are deposited and self-organized. Conversely, for cobaltNPs, eitherdispersedin hexaneordeposited on the substrate,

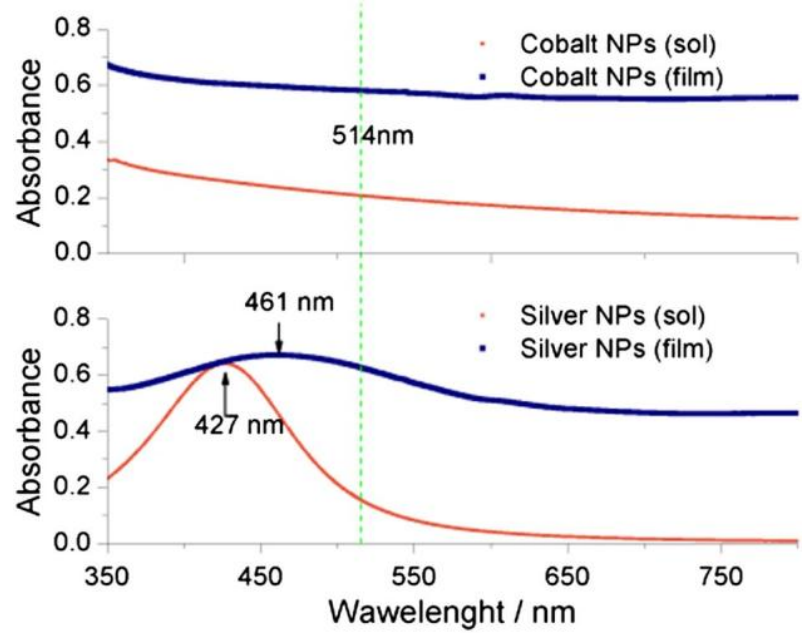

Figure 2. UV-Visible absorption spectra of cobalt (7.4-nm diameter) and silver $(5.3 \mathrm{~nm})$ NPs either dispersed in hexane (sol) or deposited in the form of 3D 'supracrystals' on silica glass plates (film). 
no particular absorption is observed. From Mie theory, the surface plasmon in pure cobalt material is expected in the UV range. However, because of high damping and interband transition, the Mie theory is recognized to be insufficient to properly evaluate the SPR wavelength for Co NPS. ${ }^{[35]}$

\section{Low wavenumber Raman studies}

Figure 3 shows a typical low wavenumber Raman spectrum obtained at room temperature for Co NPs self-organized on a silicon wafer. According to Lamb's theory ${ }^{\left[{ }^{[14]}\right.}$ the vibration modes of a free elasticsphereare described with the angular quantum momentum number I. From the selection rules, established in reference, ${ }^{[15]} \mathrm{Ra}$ man active modes are characterized by an even quantum number. The mode at the lowest wavenumber, pointed at $10 \mathrm{~cm}^{-1}$, is attributed to the quadrupolar $(I=2)$ mode while the second one, around $20 \mathrm{~cm}^{-1}$, is attributed to the breathing mode $(I=0)$. As mentioned before, the free sphere model is well documented, and no significant changes are expected when the metallic NPs are embedded in an organic medium. ${ }^{\left[{ }^{36]}\right.}$ We present the first observation for self organized cobalt NPs. The narrow size distribution and high stability against oxidation and coalescence of our Co NPs combined to the high luminosity of our spectrometer at this wavenumber range made the observation of both breathing and quadrupolar modes possible. To our knowledge, the observation of quadrupolar and breathing modes using 514.5-nm exciting laser line has never been reported in the literature for cobalt NPs. Moreover, it is commonly assumed that the coupling between the surface plasmon resonance and the confined vibrational modes of a free sphere is required to promote the Raman signature of silver or gold. Implicitly, that would signify that metals with a SPR far away from the used exciting laser line cannot give rise to observable Lamb's modes. Indeed Gangopadhyay et al. ${ }^{[37]}$ reported Raman scattering from radial breathing mode for Cobalt NPs in silica matrix using 351.1 UV laser line invoking the strong light scattering close to the SPR at this wavelength. Despite the absence of SPR in all the visible light range for cobalt NPs, a characteristic assigned to the strong damping effect arising from sand $d$ transitions, ${ }^{[38]}$ we clearly observethese vibration modes atpositions in accordance with their theoretical values.

These results are confirmed by the depolarization ratios, $I_{\mathrm{VH}} / \mathrm{I}_{\mathrm{VV}}$. In this notation, the first index stands for the polarization of the laser light, which is, in our case, always perpendicular to the light propagation direction, while the second index stands for the polarization analysis. The measured intensity ratios for the Raman active

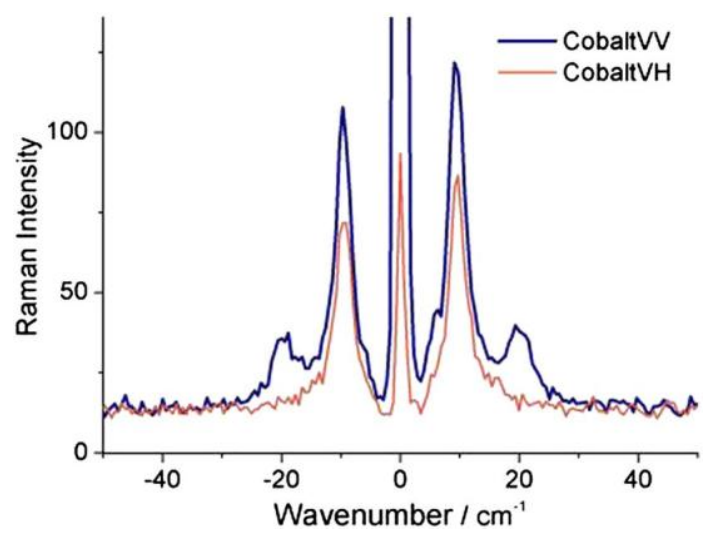

Figure 3. Polarized Raman spectra of cobalt NPs self-organized on silicon wafer. modes are respectively 0.75 and 0 , in agreement with the theoretical ratios of $3 / 4$ and 0 , respectively. ${ }^{[15,39]}$ These depolarization ratios suggest that a non-resonant Raman process ${ }^{[40]}$ is indeed responsible for the observed bands.

The wavenumber of the breathing mode, which is pointed at $19.9 \mathrm{~cm}^{-1}$, is also in accordance with previous results obtained by femtosecond pump-probe spectroscopy at short time scale on Co samples prepared with the same route, $600 \mathrm{GHz}\left(\right.$ i.e. $\left.19.8 \mathrm{~cm}^{-1}\right){ }^{[1]}$

Moreover, the wavenumber of thesemodesvis reciprocal to the particle diameter and can be approximated as ${ }^{[12,41,42]}$

$$
v_{1} 1 / 4 S_{1}: V_{1}=D: C
$$

where $V_{0}=5810 \mathrm{~m} \mathrm{~s}^{-1}$ and $V_{2}=3080 \mathrm{~m} \mathrm{~s}^{-1}$ are the longitudinal and transverse sound velocities, respectively, $\mathrm{D}$ is the average diameter of the NPs and $c$ is the light velocity in vacuum. $S_{\mid}=0.76$ is a dimensionless constant depending on the ratio of longitudinal and transverse velocities of sound within the particle as well as on the boundary conditions of the particle. ${ }^{[37]}$

The wavenumbers calculated in free sphere theoretical frame are reported in Table 1 and are in accordance with experimental values.

\section{Comparison between self-organized silver and cobalt nano-} particles_relative intensities

In order to evaluate the Raman response from different metallic NPs, we measured the relative intensity of similar modes in silver and cobalt NPs. A detailed calculation of the scattering efficiency can be done in terms of particle size distribution and light-tovibration coupling coefficient, ${ }^{[41]}$ but this is beyond the scope of this paper. Figure 4 presents Raman measurements on silver $(5.3 \mathrm{~nm})$ and cobalt $(7.4 \mathrm{~nm}) \mathrm{NPs}$, plotted in relative units. Inthelack of internal reference, precise quantitative comparison is impossible because of the strong dependency on numerous experimental factors. However, careful attention has been paid to work in strictly

Table 1. Calculated and measured positions of the Lamb' NPs modes

\begin{tabular}{llc|}
\hline Lamb' mode & $\mathrm{I}=0$ & $\mathrm{I}=2$ \\
\hline Calculation & $19.89 \mathrm{~cm}^{-1}$ & $10.54 \mathrm{~cm}^{-1}$ \\
Experimental (this study) & $19.9 \mathrm{~cm}^{-1}$ & $10.2 \mathrm{~cm}^{-1}$ \\
Pump-probe experiment ${ }^{[1]}$ & $19.8 \mathrm{~cm}^{-1}$ & Not observed \\
\hline
\end{tabular}

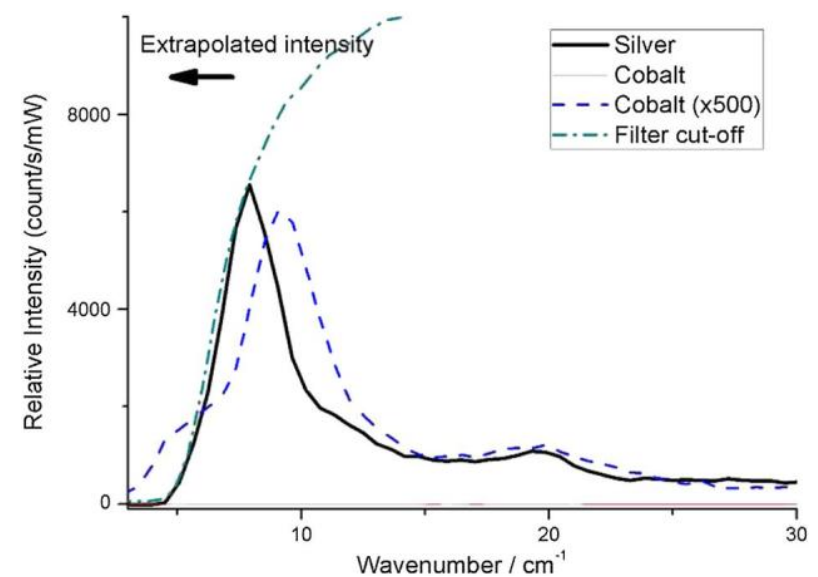

Figure4. Relative Ramanintensity of cobaltand silverNPs self-organized. 
similar conditions for the two materials, and some qualitative conclusions can be drawn.

The higher wavenumber mode, the breathing vibration of a free sphere, is the less intense of the two, but it is far enough from the Rayleigh line to avoid any distortion effect because of the filter response. This purely radial vibration is not expected to produce any significant modulation of the electric dipoles, ${ }^{[17,43]}$ and its intensity should therefore mostly depend on the intrinsic polarizability of both metals. We evaluate the efficiency ratio between the two metals to be about 500 for this mode. Comparison between the quadrupolarmodes at lowerwavenumber is more difficult because of the vicinity of the filter cut-off (dashed line), below $8 \mathrm{~cm}^{-1}$. We could not measure the intensity of this mode for silver NPs, but the black arrow in Fig. 4 shows the value extrapolated from former experiments by Courty et al. ${ }^{[21]}$ This extrapolation (not shown here) was performed by superimposing previous data in the low frequency region in which the shape of the Raman spectrum is identical to have the best match. The quadrupolar mode of silver being strongly coupled to the surface plasmon, the exaltation is expected to be more significant in the lower energy mode because of strong modulation of the electric dipole. One can approximate an enhancement of at least 3 orders of magnitude, explaining the relative easiness to experimentally observe these types of modes in silver based materials.

\section{Conclusion}

Lamb's quadrupolar and breathing modes were unexpectedly observed with green laser excitation on room temperature Raman spectra of cobalt NPs. Such observation in a metal which does not present any SPR in the visible range was made possible by taking advantage of the NPs narrow size distribution and their high stability against oxidation and coalescence. However studies below $8 \mathrm{~cm}^{-1}$ required multiple monochromators. The measured depolarization ratios support the assignment to Lamb's modes. Looking for such modes in the lack of a SPR offers a new basis to evaluate the coupling process that is responsible for the high intensity observed in metals classically used in plasmonic applications.

\section{References}

[1] D. Polli, I. Lisiecki, H. Portalès, G. Cerullo, M.-P. Pileni, ACS Nano 2011, 5, 5785-5791.

[2] Z. Yang, I. Lisiecki, M. Walls, M.-P. Pileni, ACS Nano 2013, 7, 1342-1350.

[3] G. Gouadec, Ph. Colomban, Prog. Cryst. Growth Charact. Mater. 2007, 53, 1-56.

[4] H. Ko, S. Singamaneni, V. V. Tsukruk, Small 2008, 4, 1576-1599.

[5] Z. Zhang, N. Zhang, Rare Met. 2010, 29, 561-566.

[6] V. Bakumov, K. Gueinzius, C. Hermann, M. Schwarz, E. Kroke, J. Eur. Ceram. Soc. 2007, 27, 3287-3292.

[7] R. Jiang, B. Li, C. Fang, J. Wang, Adv. Mater. 2014, 26, 5274-5309.

[8] S. Qu, Y. Gao, X. Jiang, H. Zeng, Y. Song, J. Qiu, C. Zhu, K. Hirao. J. Opt. Commun. 2003, 224, 321-327.

[9] I. Lisiecki, F. Billoudet, M. P. Pileni, J. Phys. Chem. 1996, 100, 4160-4166.

[10] Ph. Colomban, H. D. Schreiber, J. Raman Spectrosc. 2005, 36, 884-890.
[11] Ph. Colomban, J. Nano Res. 2009, 8, 109-132.

[12] A. Tamura, K. Higeta, T. Ichinokawa, J. Phys. C Solid State Phys. 1982, $15,4975$.

[13] A. Tamura, K. Higeta, T. Ichinokawa, J. Phys. C Solid State Phys. 1983, 16, 1585.

[14] N. D. Fatti, C. Voisin, F. Chevy, F. Vallée, C. Flytzanis, J. Chem. Phys. 1999, $110,11484-11487$

[15] E. Duval, Phys. Rev. B. 1992, 46, 5795-5797.

[16] E. Duval, A. Boukenter, B. Champagnon, Phys. Rev. Lett. 1986, http:// www.igm.nsc.ru/labs/lab436/ ovsyuk/images/PRL56.2052.86.pdf (accessed February 21, 2014).

[17] B. Palpant, H. Portales, L. Saviot, J. Lermé, B. Prével, M. Pellarin, E. Duval, A. Perez, M. Broyer. Phys. Rev. B. 1999, 60, 17107-17111.

[18] H. Portales, L. Saviot, E. Duval, M. Fujii, S. Hayashi, N. Del Fatti, F. Vallée. J. Chem. Phys. 2001, 115, 3444-3447.

[19] R. Carles, C. Farcău, C. Bonafos, G. Benassayag, B. Pécassou, A. Zwick, Nanotechnology 2009, 20, 355305-355311.

[20] A. Courty, I. Lisiecki, M. P. Pileni, J. Chem. Phys. 2002, 116, 8074-8078.

[21] A. Courty, A. Mermet, P. A. Albouy, E. Duval, M. P. Pileni, Nat. Mater. 2005, 4, 395-398.

[22] P. Verma, W. Cordts, G. Irmer, J. Monecke, Phys. Rev. B. 1999, 60, 57785785.

[23] R. S. Cataliotti, G. Compagnini, A. Morresi, M. Ombelli, P. Sassi, Phys. Chem. Chem. Phys. 2002, 4, 2774-2779.

[24] H. Lamb, Proc. Lond. Math. Soc. 1881, s1-13, 189-212.

[25] E. Duval, L. Saviot, A. Mermet, D. B. Murray, J. Phys. Condens. Matter 2005, 17, 3559.

[26] M. Ferrari, F. Gonella, M. Montagna, C. Tosello, J. Raman Spectrosc. 1996, 27, 793-797.

[27] A. Tanguy, J. P. Wittmer, F. Leonforte, J.-L. Barrat, Phys. Rev. B. 2002, 66, 174205.

[28] I. Lisiecki, M. P. Pileni, Langmuir2003, 19, 9486-9489.

[29] D. Parker, I. Lisiecki, C. Salzemann, M.-P. Pileni, J. Phys. Chem. C2007, $111,12632-12638$

[30] I. Lisiecki, C. Salzemann, D. Parker, P.-A. Albouy, M.-P. Pileni, J. Phys. Chem. C2007, 111, 12625-12631.

[31] I. Lisiecki, P.-A. Albouy, M.-P.Pileni, Adv. Mater. 2003, 15, 712-716.

[32] P. Aubertin, M. A. Ben Aïssa, N. Raouafi, S. Jouaret, A. Courty, E. Maisonhaute, Nano Res. 2015, 8, 1615-1626.

[33] M.-H. Lin, H.-Y. Chen, S. Gwo, J. Am. Chem. Soc. 2010, 132, 11259-11263.

[34] A. Courty, J. Phys. Chem. C2010, 114, 3719-3731.

[35] ž. Kaminskienè, I. Prosyčevas, J. Stonkutè, A. Guobienè, Acta Phys. Pol. A. $2013,123,111$.

[36] D. B. Murray, L. Saviot, Phys. Rev.B. 2004, 69,094305-094313.

[37] P. Gangopadhyay, T. R. Ravindran, K. G. M. Nair, S. Kalavathi, B. Sundaravel, B. K. Panigrahi, Appl. Phys. Lett. 2007, 90, 063108-063111.

[38] V. G. Kravets, A. K. Petford-Long, X. Portier, L. V.Poperenko, M. Kolesnik, J. Magn. Magn. Mater. 2000, 217, 129-138.

[39] G. Mariotto, M. Montagna, G. Viliani, E. Duval, S. Lefrant, E. Rzepka, C. Maï, EPL Europhys. Lett. 1988, 6, 239.

[40] L. Saviot, D. B. Murray, M. del C. M. de Lucas, Phys. Rev. B. 2004 69, 113402.

[41] M. Ivanda, K. Furić, S. Musić, M. Ristić, M. Gotić, D. Ristić, A. M. Tonejc, I. Djerdj, M. Mattarelli, M. Montagna, F. Rossi, M. Ferrari, A. Chiasera, Y. Jestin, G. C. Righini, W. Kiefer, R. R. Gonçalves. J. Raman Spectrosc. 2007, 38, 647-659.

[42] M. Montagna, R. Dusi, Phys. Rev. B. 1995, 52, 10080-10089.

[43] J. I. Gersten, D. A. Weitz, T.J. Gramila, A. Z. Genack, Phys. Rev. B. 1980, 22, 4562-4571.

\section{Supporting information}

Additional supporting information may be found in the online version of this article at the publisher's web site. 